\title{
Hallesche Beiträge
}

\section{zur Europäischen Aufklärung}

Schriftenreihe des Interdisziplinären Zentrums

für die Erforschung der Europäischen Aufklärung

Martin-Luther-Universität Halle-Wittenberg 



\section{Die Kantate als Katalysator}

Zur Karriere eines

musikalisch-literarischen Strukturtypus

um und nach 1700

Herausgegeben von

Wolfgang Hirschmann und Dirk Rose

De Gruyter 
Herausgeber:

Thomas Bremer, Daniel Cyranka, Elisabeth Décultot, Jörg Dierken, Robert Fajen, Daniel Fulda, Frank Grunert, Wolfgang Hirschmann, Yvonne Kleinmann, Heiner F. Klemme, Andreas Pečar, Jürgen Stolzenberg, Heinz Thoma, Sabine Volk-Birke

Wissenschaftlicher Beirat:

Anke Berghaus-Sprengel, Albrecht Beutel, Ann Blair, Michel Delon, Avi Lifschitz, Robert Louden, Laurenz Lütteken, Brigitte Mang, Steffen Martus, Laura Stevens

Redaktion: Andrea Thiele

Gedruckt mit freundlicher Unterstützung der Fritz Thyssen Stiftung.

Fritz Thyssen Stiftung

ISBN 978-3-156936-0

e-ISBN (PDF) 978-3-11-057281-0

e-ISBN (EPUB) 978-3-11-057135-6

ISSN 0948-6070

Library of Congress Control Number: 2018934807

Bibliografische Information der Deutschen Nationalbibliothek

Die Deutsche Nationalbibliothek verzeichnet diese Publikation in der Deutschen Nationalbibliografie; detaillierte bibliografische Daten sind im Internet über http://dnb.dnb.de abrufbar

(C) 2018 Walter de Gruyter GmbH, Berlin/Boston

Satz: Dörlemann Satz, Lemförde

Druck: CPI books GmbH, Leck

@ () Gedruckt auf säurefreiem Papier

Printed in Germany

www.degruyter.com 